\title{
Las tesis doctorales de la FACPYA: Análisis bibliométrico de sus referencias (2011-2015) (The doctoral theses of the FACPYA: Bibliometric analysis of their references [2011-2015])
}

\section{'José Segoviano Hernández iiJosé Daniel Olivares Márquez}

\begin{abstract}
This article aims to present the partial results of a larger bibliometric study in which the quality of the bibliographical references of the doctoral theses of the Universidad Autónoma de Nuevo León is analyzed, in this partial study, the trends of citation of the two PhD's degree of FACPYA in the field of Administration and Accounting are analyzed, taking as a field of study 35 doctoral theses from both areas, defended during the years 2011 to 2015 and which are in the databases from the UANL Digital Collection. For the analysis, 5 bibliometric index variables were used at international level to evaluate scientific publications through which it was proposed to answer the following questions: What is the average of the references that contain these documents? What kind of documents do they use? How old are the cited documents? (Price index), Which language is the most used?, Which percentage presents errors in the application of the bibliographic style?. The objective of the work was to identify the type of sources that were consulted to elaborate the theses. The important findings about the average number of documents was acceptable, as well as the varied type of documents used, as is the case with most of the studies in the subject. The index of references of journal articles indicates an acceptable consumption use of scientific information, the Price Index describes acceptable percentages of updated information consumption and identified errors in the handling of the bibliographic style.
\end{abstract}

Key words: bibliometric study, doctoral thesis, obsolescence of literature, price index, references. JEL: C490, C180, Y400.

Resumen. El presente artículo tiene como propósito presentar los resultados parciales de un estudio bibliométrico mayor en el que se analiza la calidad de las referencias bibliográficas de las tesis de doctorado de la Universidad Autónoma de Nuevo León, en este estudio parcial se analizan las tendencias de citación de las tesis de dos Doctorados de la FACPYA, en el campo de la Administración y la Contaduría, tomando como población de estudio 35 tesis doctorales de ambos campos, defendidas durante los años 2011 a 2015 y que se encuentran en la base

\footnotetext{
' Facultad de Ciencias Políticas y Relaciones Internacionales (UANL), Praga y Trieste s/n Col. Residencial Las Torres, Monterrey, Nuevo León, México. C.P. 64930. Email: jose_segoviano01@hotmail.com

ii Dirección de Bibliotecas (UANL), Av. Alfonso Reyes \#4000 nte. Col Regina C.P. 64290, Monterrey, Nuevo León, México. Email: redolivares@hotmail.com
} 
de datos Colección Digital UANL. Para el análisis se determinaron 5 variables de indicadores bibliométricos de índices empleados a nivel internacional para evaluar publicaciones científicas a través de las cuales se planteó contestar a los siguientes cuestionamientos: ¿Cuál es la media de las referencias que contienen estos documentos? ¿Qué tipo de documentos utilizan? ¿Qué edad tienen los documentos citados? (índice Price) ¿Qué idioma es el más utilizado? ¿Qué porcentaje presenta errores en la aplicación del estilo bibliográfico?. El objetivo del trabajo fue identificar el tipo de fuentes que fueron consultadas para elaborar las tesis. Los hallazgos importantes sobre la media de la cantidad de documentos resultaron aceptables, así como el variado tipo de documentos utilizados, como es el caso de la mayoría de los estudios sobre el tema. El índice de referencias de los artículos de revistas indica aceptable consumo de información científica, el Índice Price describe porcentajes aceptables de consumo de información actualizada y se identificaron errores en el manejo del estilo bibliográfico.

Palabras clave: estudio bibliométrico, índice de price, obsolescencia de la literatura, referencias, tesis doctoral.

\section{Introducción}

Uno de los principales requisitos para la culminación de los estudios de doctorado es la elaboración y defensa de la tesis que se derive de una investigación. Por ello, se considera que una tesis doctoral es una "contribución especial al conocimiento, que justifica su originalidad porque presencia el descubrimiento de hechos nuevos y/o derivados del ejercicio de una crítica independiente del autor" (Rivera, 2011, p. 22).

En este sentido García (1998) sostiene que el objetivo de la tesis es mostrar las nociones indispensables para desarrollar un proyecto de investigación, el cual implica elementos teóricos para sustentar el trabajo escrito, y en esto último las citas desempeñan una función crucial en el discurso del escrito en donde "a través de la citación, se conforman cuerpos compartidos de literatura disciplinar, extensas redes de comunicación científica y complejos vínculos de cooperación intelectual entre los investigadores" (González de Requena, Andana y Duhart, 2015, p. 182).

Las investigaciones generadas de tesis doctorales proporcionan una relevancia a las universidades por el impacto de sus estudios y resultados. En palabras de Ortiz, González, González, y Infante (2010) "las tesis doctorales constituyen salidas importantes que favorecen el mayor y más rápido impacto de los resultados de investigación" (p. 82), beneficiando la visibilidad y la calidad de los programas de estudio de las universidades. 
El Programa Sectorial de Educación, manifiesta que "los estudios de posgrado son la ruta para la formación de los recursos humanos altamente especializados requeridos para atender las necesidades de las instituciones de educación superior, centros de investigación, organismos de gobierno y empresas" (SEP, 2013, p. 33). En años recientes, de acuerdo con Torres, Ruiz y Meza (2017), "la expansión de los posgrados en México y las políticas de Estado en relación con una educación superior de calidad constituye un nuevo reto para las instituciones públicas y privadas que ofertan programas de posgrado" (p.7).

Sánchez (2008) clasifica a los doctorados en dos tipos y los caracteriza considerando que, "los programas conducentes al Ph. D. o Doctorado en Ciencias enfatizarán la investigación y la docencia, mientras los programas conducentes a doctorados profesionales enfatizarán el desarrollo de modelos o pautas apropiadas a la consultoría y la prestación de servicios" (p. 333), y en el caso específico del doctorado en su formato de Doctorado en Filosofía (Ph. D.) o Doctorado en Ciencias, describe que:

Constituye el subnivel superior de los estudios de postgrado, siendo su fin principal el de una profundización tal que se lleve a explorar y entrar en conocimiento de las fronteras de desarrollo del saber, con dominio de la metodología de investigación científica o de las capacidades de producción intelectual o creación artística demostradas por medio de una contribución original en un campo dado de la ciencia, la tecnología, las artes o las humanidades. (Sánchez, 2008, p. 333)

En nuestro país, el Consejo Nacional de Ciencia y Tecnología (CONACYT) es quien evalúa de manera rigurosa, tanto cualitativa como cuantitativa los programas de posgrado, donde están incluidos los doctorales, y lo hace a través del Programa Nacional de Posgrados de Calidad (PNPC), su propósito es reconocer a los programas que cuentan con núcleos académicos básicos, altas tasas de graduación, infraestructura necesaria y alta productividad científica o tecnológica, lo cual les permite lograr la pertinencia de su operación y óptimos resultados. Para ello, establece una serie de criterios de evaluación que los programas doctorales, deben de cumplir para considerarlos programas de calidad y aplica diversos indicadores como la productividad académica, la movilidad nacional e internacional y la pertenencia a grupos de investigación. Lo que posteriormente permite una articulación con el Sistema Nacional de Investigadores (SNI) a los 
académicos, estudiantes y egresados, para desarrollar como eje de su trabajo la investigación, individual y en grupo (Jiménez, 2014).

El PNPC, cuenta con el Padrón del Programa Nacional de Posgrados de Calidad (PPNPC), y a través de su página, mantiene un sistema de consultas, donde ofrece variada información descriptiva de estos programas, como son matriculas por áreas de conocimiento y entidad federativa, entre otros. Sin embargo carece de estrategias de información y de retroalimentación a las comunidades académicas en relación con la cantidad, calidad e impacto de las tesis doctorales.

Este hecho es importante porque como describen Buela-Casal y García-Berro, citados por Musi-Lechuga (2011), la productividad científica supone uno de los conceptos más importantes para los profesores, programas de doctorado y para las universidades a la hora de recibir financiamiento y también por lo expresado por Núñez Jover, citado por Sánchez (2008), que "si se revisan los programas de postgrado que se imparten en América Latina, se verá que muchos de ellos se parecen bastante a la enseñanza de pregrado por su excesiva escolaridad y poca importancia asignada al trabajo de producción intelectual" (337).

Estas reglas y transformaciones han repercutido en los doctorados, así como en sus circuitos de comunicación científica, donde la publicación de los resultados en revistas, se ha convertido en una obligación científica (Retamozo, 2014), esto "supone grandes retos para los investigadores y para las instituciones donde se genera el conocimiento" (p. 10). Teniendo en cuenta estas necesidades, cabe señalar que las revistas observan variables que pueden medirse cuantitativamente a través de estudios bibliométricos y que ellos proporcionan resultados de interés, al respecto tanto, para los autores como para los editores de las revistas.

En este contexto, el Doctorado en Contaduría y el Doctorado en Filosofía con especialidad en Administración, de la UANL, cuentan con adscripción al PNPC, y de los cuales no se encontraron publicaciones ni estudios bibliométricos que analicen sus tesis de doctorado. Lo que nos lleva a los siguientes cuestionamientos:

¿Cuál es la media de las referencias que contienen estos documentos?

¿Qué tipo de documentos utilizan?

¿Qué edad tienen los documentos citados? (índice Price)

¿Qué idioma es el más utilizado?

¿Qué porcentaje presenta errores en la aplicación del estilo bibliográfico? 
El propósito de este artículo es mostrar los resultados parciales de un estudio mayor sobre la calidad de las referencias bibliográficas de las tesis de doctorado de la UANL. En la presente investigación se analizan, a través de 5 indicadores bibliométricos, los resultados de 35 tesis de ambos doctorados, defendidas entre los años 2011 y 2015, con el objetivo de identificar el tipo de fuentes que fueron consultadas para elaborar las tesis y obtener una visión sobre el estado de sus referencias, e identificar patrones implícitos en su proceso de citación en este período de tempo y con ello proponer indicadores bibliométricos que permitan su mejora y su evaluación.

\section{Fundamentos teóricos}

El objetivo de la bibliometría es el tratamiento y estudio de datos cuantitativos procedentes de las publicaciones científicas, "en la actualidad, los indicadores bibliométricos o de producción científica se aceptan como un indicador válido de los resultados de la investigación, junto a otros indicadores como patentes 0 nuevos productos en las áreas más tecnológicas" (Bordons y Zuluetab, 1999, p. 791). No obstante que el análisis de las referencias no está exento de críticas, es uno de los indicadores más utilizados y más problemáticos, en la investigación cienciométrica (Vallejo, Fernández y Torralbo, 2006). En los estudios bibliométricos cabe distinguir entre cita, que es el reconocimiento que un documento recibe de otro, y referencia bibliográfica, que es el reconocimiento que un documento da a otro (Price, citado por Pulgarín, Carapeto y Cobos, 2004).

El proceso de citar es "mencionar las palabras o las ideas de una persona. Esas palabras o ideas, al ser escritas o dichas por un autor se constituyen en una cita" (Patiño, 2007, p. 19). Y respecto a las referencias bibliográficas, para algunos, estas "constituyen la manifestación material del enlace que existe entre la labor de investigación que se expone y la labor de investigación precedente, y son un conjunto mínimo de datos que permite la identificación de una publicación o de una parte de ella" (Silva, Rodríguez y del Campo, 2013, p. 85), e indican al lector de quién es la cita que está leyendo y donde puede encontrarla en la fuente original. Por ello, cada referencia bibliográfica "debe ser veraz (completa y exacta) tanto en sus elementos formales de citación como en su contenido" (Ponce y Rodríguez, citado por Osca, Civera, y Peñaranda, 2009, p. 300) y para cumplir estos cometidos las referencias bibliográficas deben ser pertinentes y exactas, con el fin de acceder a la versión original. 
Las consecuencias de estos errores es que impiden localizar el documento original, lo que perjudica al autor y a las revistas, "ya que traen como consecuencia que las citaciones que reciben algunos de los trabajos publicados en revistas recogidas en alguna bases de datos, como, por ejemplo, las del ISI, se pierdan, siendo el número de citaciones un elemento funda-mental para el cálculo de algunos indicadores bibliométricos, como es el factor de impacto de las revistas" (Osca, Civera, y Peñaranda, 2009, p. 301).

Urbizagástegui y Restrepo citados por Meneses, Romero y Sesma (2013), sostienen que el análisis de citas "describe las características de los documentos referenciados identificando revistas más frecuentemente mencionadas; tipos de documentos, edad de la literatura, autores más frecuentemente citados, idiomas empleados en las referencias (p. 36).

En una investigación la mayor concentración de las citas está en la fundamentación teórica, lo que lleva a cuestionar sobre cuántas referencias deben tener un marco teórico de una tesis de doctorado, al respecto, Hernández, Fernández y Bautista (2010), consideran que, aun y cuando no son estándares, sino que resultan de la mayoría de los casos, ellas generalmente contienen entre $65 \mathrm{y}$ 120 , mientras que las de maestría entre 30 y 40 y un artículo de revista científica entre 50 y 70 .

Las tesis de doctorado, de acuerdo con Urbano (2000), contienen una mayor variedad y cantidad de los documentos citados, a diferencia de las revistas, que tiene una tendencia hacia recoger citas de otros artículos de revistas y como apuntan Vallejo, Fernández y Torralbo, (2006) existe una estrecha relación entre las tesis doctorales y las referencias, debido a que "una tesis doctoral debe realizar una completa y ordenada revisión de la literatura, tanto conceptual como de investigación, que exponga los hallazgos más recientes" (p. 384).

Es una práctica común en los evaluadores de artículos o proyectos de investigación soliciten, en base al «Índice de Price», referencias con antigüedad no mayor a cinco años. Este índice es uno de los métodos más empleados al realizar un estudio Bibliométrico, hace referencia a la obsolescencia de la información científica, al constatarse que la literatura científica pierde actualidad cada vez más rápidamente. En opinión de Oyola, Soto y Quispe (2014), respecto a la exigencia de la actualidad de las citas, en las publicaciones periódicas (revistas) y especialmente en las tesis, la consideran una "práctica sin sustento y negativa, para quienes desarrollan investigación innovadora o en campos donde las publicaciones son escasas" (p. 381). 
Por otra parte, el doctorando, en la elaboración de su tesis, requiere seguir lineamientos específicos, proporcionados por la institución que le permitan desarrollar de acuerdo a los mismos su proyecto de investigación. Sin embargo, en palabras de de-Miguel (2010), esto no se suele cumplir eficientemente:

Los reglamentos establecidos por las distintas universidades sobre los programas de doctorado y las tesis suelen incidir más sobre las cuestiones administrativas que sobre las técnicas... Tampoco suelen precisar los criterios específicos que deberán tener en cuenta para valorar la calidad de una tesis. (p. 2)

La evaluación del impacto científico de las tesis doctorales es una necesidad perentoria debido a su pertinencia y a las demandas actuales para contribuir en la formación de las nuevas generaciones, en las condiciones sociales actuales (Ortiz, González, González, y Infante Pérez, 2010). Para ello es "necesario efectuar una evaluación a posteriori sobre las tesis con el fin de estimar su contribución real al conocimiento científico y a la resolución de problemas prácticos, para lo cual resulta necesario efectuar un seguimiento a lo largo del tiempo de las aportaciones y aplicaciones derivadas de la misma mediante publicaciones, patentes, protocolos, ponencias y proyectos." (de-Miguel, 2010, Sección 4, párr. 1).

En el área de la bibliometría se han realizado estudios, con la finalidad de analizar el tipo de referencias utilizadas en la elaboración de tesis a nivel doctorado. Los resultados encontrados por Vallmitjana (2008), en 26 tesis de doctorado enfocadas a las ciencias químicas donde analizó las publicadas de los años 1995-2003, encontró que el 79 \% utilizaba artículos y el $50 \%$ de ellos no era mayor a nueve años.

Un estudio similar (Vallejo, Fernández y Torralbo, (2006), revisaron 241 tesis doctorales de Educación Matemática, con una cobertura de 1975 a 2002, encontró que las tesis contenían un promedio de 200 referencias por tesis; también se destacó que el idioma inglés fue el más utilizado seguido del español y el tipo de fuente fueron los libros, sobre la antigüedad, el promedio fue de 12 años; así también indicó que se encontraron errores y distorsiones en las citas registradas.

Meneses, Romero, y Sesma (2013), en su estudio analizaron una muestra de 172 tesis de maestría de la Universidad Veracruzana, encontraron que para estas tesis, elaboradas entre 1992 y el año 2010; en promedio tienen 22 referencias, con un máximo de 38 fuentes consultadas y un mínimo de 6 . La edad promedio es de 8.53 años. El tipo de fuentes consultadas, en un $60.5 \%$ 
son libros, seguidas por revistas, con un $14 \%$. También se consultan sitios de Internet, manuales, leyes y tesis, entre otros documentos. Con relación al idioma, el $86.6 \%$ de las fuentes, está en español y aproximadamente el $13 \%$ en inglés, el $1 \%$ restante, en otro idioma. Urbano (2000), a través de un análisis de citas en 54 tesis doctorales de informática, de la Universitat de Politécnica de Calalunya, encontró una elevada obsolescencia de la bibliografía citada, "ya que una de las características de la investigación llevada a cabo por los doctorandos acostumbra a ser la revisión de la bibliografía sobre un tema considerando sus fundamentos y los primeros desarrollos sobre el mismo" (sección de Conclusiones, párr. 2).

Brito, Ladrón de Guevara y Rosas (2010), analizaron 35 tesis de doctorado del año 2009 de los programas de Ciencias Biológicas, Ciencias Biomédicas y Ciencias Químicas, de la UANM, de las que obtuvieron 5683 citas, lo cual da en promedio 140 referencias por tesis. Los documentos más citados fueron los artículos científicos, seguidos de las monografías, y en menor porcentaje las tesis, patentes, actas de conferencias, reportes y recursos electrónicos. El idioma más citado fue el inglés, en su mayoría los artículos más citados tienen una edad de cinco a nueve años.

\section{Datos y metodología}

El diseño de este estudio es descriptivo y censal, mediante análisis de documentos y por su temporalidad, se considera un estudio longitudinal de indicadores bibliométricos. El análisis bibliométrico de la bibliografía citada en las tesis doctorales, es un método que no interfiere en el comportamiento de los doctorandos mientras que se toman los datos, además de que permite una disponibilidad relativamente sencilla de los mismos (Urbano, 2000).

Para la recolección de las tesis se realizó su búsqueda en la Base de Datos Colección Digital de la UANL «http://cd.dgb.uanl.mx/», la cual acopia las tesis doctorales defendidas en la UANL desde el año 2011. Se identificaron y se acopiaron las 35 tesis (10 del Doctorado en Contaduría y 25 del Doctorado en Administración). Una vez reunidos los documentos se ingresó a cada uno de ellos para recuperar el listado de referencias y elaborar los análisis descriptivos. En la recolección de datos participaron tres investigadores y 3 colaboradoras de prácticas profesionales. Para la organización de los datos, se registraron primero en Excel y posteriormente se pasaron a SPSS versión 19, con el fin de agrupar los datos y realizar los análisis estadísticos propuestos. 
Se identificaron y cuantificaron 5 variables 0 indicadores, con el fin de responder las preguntas del estudio: 1) Media de las referencias, 2) Tipo de documentos, 3) Actualidad de las referencias «Índice Price», 4) Idioma, y 5) Errores en la descripción de las referencias.

\section{Resultados}

La muestra de las 35 tesis analizadas se presenta en la Tabla 1, contiene las frecuencias de las tesis defendidas por año, donde sobresalen los años 2012 y 2014 , en los que se presentaron 8 tesis respectivamente y en donde se concentró el $(45.7 \%)$ de las tesis registradas.

En la Figura 1 se puede observar que en el año 2014, mientras que el doctorado en Administración alcanza el nivel más alto, con 7 tesis presentadas, en contraste el Doctorado en Contaduría desciende al nivel más bajo con sólo una tesis.

Tabla 1. Tesis presentadas por año y porcentaje sobre el total

\begin{tabular}{ccccccc}
\hline & \multicolumn{2}{c}{ Contaduría } & \multicolumn{3}{c}{ Administración } & \multicolumn{2}{c}{ Total } \\
año & $\mathrm{fr}$ & $(\%)$ & $\mathrm{fr}$ & $(\%)$ & $\mathrm{fr}$ & $(\%)$ \\
\hline 2001 & 2 & $(20.0)$ & 4 & $(16.0)$ & 6 & $(17.1)$ \\
2012 & 3 & $(30.0)$ & 5 & $(20.0)$ & 8 & $(22.9)$ \\
2013 & 2 & $(20.0)$ & 5 & $(20.0)$ & 7 & $(20.0)$ \\
2014 & 1 & $(10.0)$ & 7 & $(28.0)$ & 8 & $(22.9)$ \\
2015 & 2 & $(20.0)$ & 4 & $(16.0)$ & 6 & $(17.1)$ \\
Total & 10 & $(100.0)$ & 25 & $(100.0)$ & 35 & $(100)$ \\
\hline
\end{tabular}

Nota. $\mathrm{fr}=$ frecuencia. Elaboración propia. 
Figura 1. Evolución anual de la producción de tesis doctorales.

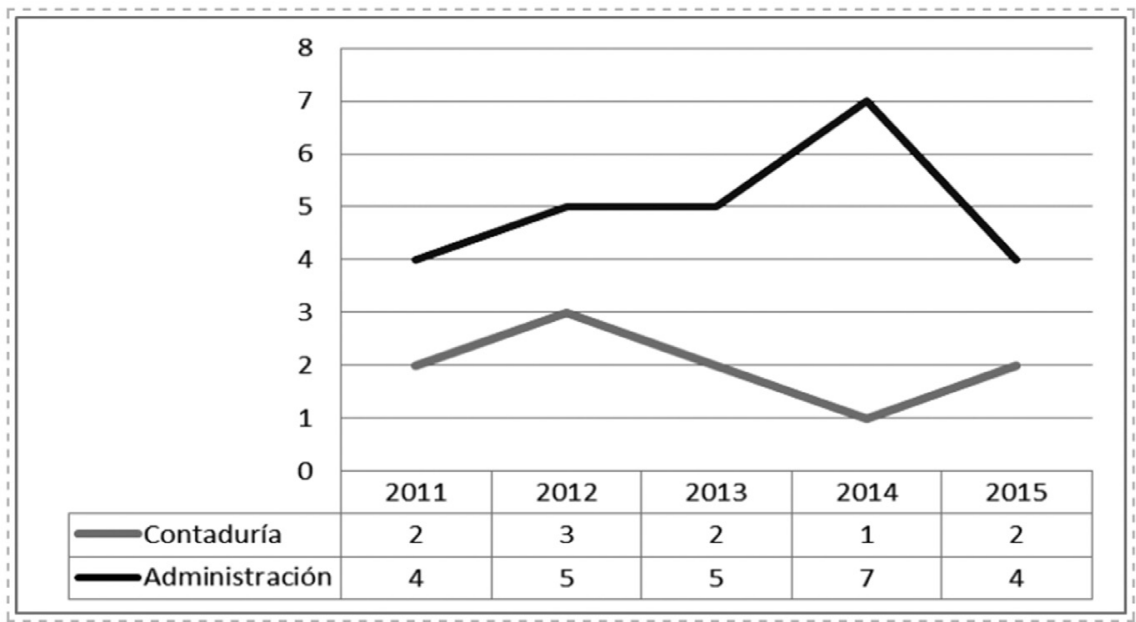

Elaboración propia.

La Tabla 2 describe el género de los sustentantes, resultando el género masculino el más representativo con el $80 \%$ y que se aprecia de igual forma en ambos doctorados.

Tabla 2. Trabajos elaborados por género del autor y por porcentaje sobre el total

Total

Contaduría

Administración

\begin{tabular}{ccccccc} 
Género & fr & $(\%)$ & fr & $(\%)$ & fr & $(\%)$ \\
\hline Mujer & 7 & $(20)$ & 2 & $(5.7)$ & 5 & $(14.3)$ \\
Hombre & 28 & $(80)$ & 8 & $(22.9)$ & 20 & $(57.1)$ \\
Total & 35 & $(100)$ & 10 & $(28.6)$ & 25 & $(71.4)$ \\
\hline
\end{tabular}

Nota. fr= Frecuencia. Elaboración propia.

¿Cuál es la media de las referencias que contienen estos documentos?

En las 35 tesis analizadas, se obtuvieron 3,325 referencias. La media de las referencias de las tesis se describe en la Tabla 3, resultando la media general de 95 referencias por tesis, y en lo individual en ambos doctorados no se observan diferencias notables, no así en el nivel máximo donde sobresale una tesis del Doctorado en Contaduría con 221 referencias, y por otra parte en el doctorado en Administración se identificó a la tesis con el menor número de referencias con 34 . 
Tabla 3. Media y desviación típica de las referencias

\begin{tabular}{ccccccc}
\hline Doctorado & Total & $(\%)$ & Media & Mínimo & Máximo & Desv. típica \\
\hline Contaduría & 979 & $(32.69)$ & 97.9 & 53 & 221 & 49.312 \\
Administración & 2346 & $(67.3)$ & 93.8 & 34 & 184 & 42.331 \\
Total & 3325 & $(100)$ & 95 & 34 & 221 & \\
\hline
\end{tabular}

Nota. Elaboración propia.

¿Qué tipo de documentos utilizan?

Centrándonos en los diferentes tipos de documentos, la tabla 4 presenta las frecuencias de los documentos referenciados, en ella se puede apreciar que los artículos de revista fueron los más consumidos con un total general de $44.6 \%$ que se observa similar en ambos doctorados, respecto a los libros en el Doctorado en Administración se aprecia un mayor porcentaje de su uso con $33.9 \%$, mientras que el Doctorado en Contaduría solo consumió un $19.2 \%$ de ellos respecto al total de documentos utilizados.

Tabla 4. Tipos de documentos utilizados

\begin{tabular}{ccccc}
\hline & \multicolumn{2}{c}{ Contaduría } & \multicolumn{2}{c}{ Administración } \\
Tipo de referencia & $\mathbf{f r}$ & $(\%)$ & fr & (\%) \\
\hline Libros & 209 & $(19.2)$ & 758 & $(33.9)$ \\
Artículos de revista & 492 & $(45.3)$ & 990 & $(44.2)$ \\
Página web & 243 & $(22.4)$ & 451 & $(20.2)$ \\
Tesis & 17 & $(1.6)$ & 80 & $(3.6)$ \\
Congresos & 8 & $(0.7)$ & 48 & $(2.1)$ \\
Leyes & 9 & $(0.8)$ & 5 & $(0.2)$ \\
Patentes & 0 & $(0.0)$ & 4 & $(0.2)$ \\
DOF & 0 & $(0.0)$ & 7 & $(0.3)$ \\
Entrevistas & 1 & $(0.1)$ & 1 & $(0.0)$ \\
Normas & 0 & $(0.0)$ & 1 & $(0.0)$ \\
Clase & 0 & $(0.0)$ & 1 & $(0.0)$ \\
Total & 979 & 100.0 & 2346 & 100 \\
\hline
\end{tabular}

Nota: Congresos= Ponencias, conferencias/ DOF= Diario Oficial de la Federación/Entrevistas= personales, de televisión/ Página Web (reportes técnicos, organizaciones como OECD, INEGI, Secretaría de Economía)/ Clase= reporte de actividad áulica. 
¿Qué edad tienen los documentos citados? (índice Price)

El índice Price que se refiere a la actualidad de los documentos referenciados, se muestra en la Tabla 5. En esta tabla se observa el número de referencias por tipo de documento, y la cantidad de referencias con antigüedad menor a 5 años respectivamente y el índice de Price.

El resultado es muy variable, por lo general un índice de Price bajo, a excepción de las referencias de las Páginas Web, donde es bastante alto. No así en los artículos de revistas donde sólo se obtuvo el $32.9 \%$ en el Doctorado en Contaduría y el 30.9 \% en el Doctorado en Administración. El índice de Price del total de las referencias del Doctorado en Contaduría fue de $36.9 \%$ y el del Doctorado en Administración fue de $31.9 \%$

Tabla 5. Actualidad de las referencias

\begin{tabular}{cccccccc}
\hline & & \multicolumn{3}{c}{ Contaduría } & \multicolumn{3}{c}{ Administración } \\
Renos & Índice & & Menos & Índice \\
Referencias & $\begin{array}{c}\text { Total } \\
\text { general }\end{array}$ & Total & $\begin{array}{c}\text { de 5 } \\
\text { años }\end{array}$ & $\begin{array}{c}\text { Price } \\
(\%)\end{array}$ & Total & $\begin{array}{c}\text { de 5 } \\
\text { años }\end{array}$ & $\begin{array}{c}\text { Price } \\
(\%)\end{array}$ \\
\hline $\begin{array}{c}\text { Libros } \\
\text { Artículos }\end{array}$ & 967 & 209 & 49 & $(23.4)$ & 758 & 144 & $(19.0)$ \\
de revistas \\
$\begin{array}{c}\text { Páginas } \\
\text { web }\end{array}$ & 1482 & 492 & 162 & $(32.9)$ & 990 & 301 & $(30.4)$ \\
$\quad$ Tesis & 97 & 243 & 134 & $(55.1)$ & 451 & 245 & $(54.3)$ \\
$\begin{array}{c}\text { Congresos } \\
\text { Total }\end{array}$ & 56 & 17 & 6 & $(35.3)$ & 80 & 36 & $(45.0)$ \\
\hline
\end{tabular}

Nota. El índice Price se calculó estimando los 5 años anteriores al año de defensa de la tesis.

Fuente: Elaboración propia.

¿Qué idioma es el más utilizado?

La distribución de las frecuencias de los idiomas expresados en las referencias bibliográficas puede ser observada en la tabla 6 , en ella se aprecia al inglés como el idioma más utilizado, con un total de 1,992 de las 3,325 referencias totales, que le asignan un $59.9 \%$, tanto en lo general como en lo individual en ambos doctorados. 
Tabla 6. Idioma de las referencias

\begin{tabular}{ccccccc}
\hline & \multicolumn{2}{c}{ Contaduría } & \multicolumn{2}{c}{ Administración } & \multicolumn{2}{c}{ Total } \\
& $\mathrm{Fr}$ & $(\%)$ & $\mathrm{Fr}$ & $(\%)$ & $\mathrm{Fr}$ & $(\%)$ \\
\hline Español & 392 & $(40.0)$ & 926 & $(39.5)$ & 1318 & $(39.5)$ \\
Inglés & 586 & $(59.9)$ & 1406 & $(59.9)$ & 1992 & $(59.9)$ \\
Italiano & 1 & $(0.1)$ & 5 & $(0.2)$ & 6 & $(0.2)$ \\
Francés & 0 & $(0.0)$ & 6 & $(0.3)$ & 6 & $(0.2)$ \\
Portugués & 0 & $(0.0)$ & 2 & $(0.1)$ & 2 & $(0.1)$ \\
Alemán & 0 & $(0.0)$ & 1 & $(0.0)$ & 1 & $(0.0)$ \\
& 979 & $(100)$ & 2346 & $(100)$ & 3325 & $(100)$ \\
\hline
\end{tabular}

¿Qué porcentaje presenta errores en la aplicación del estilo bibliográfico?

Respecto a los errores visibles en las referencias bibliográficas, la tabla 7, contiene las frecuencias de los índices de cumplimiento con el formato APA, los resultados indican altos porcentajes de estos errores ya que sólo en 5 de las 35 tesis ( 3 de del Doctorado en Contaduría y 2 de Doctorado en Administración) presentaron un cumplimiento superior al $75 \%$ en sus referencias.

Tabla 7. Frecuencia y rango de cumplimiento de las tesis en el estilo bibliográfico

Frecuencia de rango de cumplimiento en APA

\begin{tabular}{ccccccccccccc} 
& \multicolumn{3}{c}{$\begin{array}{c}\text { Referencias } \\
\text { sin }\end{array}$} & $0 \%$ a $25 \%$ & $26 \%$ a $50 \%$ & $51 \%$ a $75 \%$ & $76 \%$ a $100 \%$ & Total \\
Doctorado & $\begin{array}{c}\text { fecha } \\
\text { Total }\end{array}$ & fr & (\%) & fr & $(\%)$ & fr & $(\%)$ & fr & (\%) & Tesis \\
\hline Contaduría & 8 & 979 & 2 & $(20)$ & 4 & $(40)$ & 1 & $(10)$ & 3 & $(30$ & 10 \\
Administración & 48 & 2346 & 13 & $(52)$ & 6 & $(24)$ & 3 & $(12)$ & 2 & $(8)$ & 25 \\
Total & 56 & 3325 & 15 & $(42.9)$ & 10 & $(28.6)$ & 4 & $(11.4)$ & 5 & $(14.2)$ & 35 \\
\hline
\end{tabular}

\section{Conclusiones}

No cabe duda que el interés de tener una visión del estado de las referencias en las tesis doctorales puede ayudar a los doctorandos y a sus directores de tesis, para tener cifras comparativas que les permitan establecer parámetros para la elaboración de estos documentos. Cabe señalar que el propósito de este estudio no permite evaluar la calidad de las tesis, sino únicamente realizar un análisis de 
los documentos consultados en su elaboración, considerando índices empleados a nivel internacional para evaluar publicaciones científicas hacia donde se enfoca la elaboración de las tesis doctorales, ello con el fin de identificar fortalezas y debilidades que permitan la mejora de los doctorados de la UANL, por lo menos en lo referente las referencias bibliográficas. Los resultados obtenidos permiten concluir que la media de la cantidad de documentos es aceptable, así como el variado tipo de documentos utilizados, como es el caso de la mayoría de los estudios sobre el tema.

El índice de referencias de los 1,482 artículos de revistas indica un alto consumo de información científica y en alto porcentaje en idioma inglés, que es el preferente en la investigación científica, pero por otra parte, el bajo valor del Índice Price describe una elevada obsolescencia de la información consultada, lo que nos lleva a analizar la aplicación de medidas de mejora de este indicador, así como también, en la observación de la correcta aplicación del estilo bibliográfico dado que se detectó gran cantidad de errores y omisiones en las referencias, y que en general, no se observó apego en las normas para la redacción de las citas bibliográficas que describe la American Psychological Association.

Los hallazgos de este estudio pueden ayudar a los directores de tesis en orientar a sus doctorandos para observar parámetros bibliométricos en los diseños de los proyectos y de igual forma a los miembros de los comités evaluadores. A la administración de los posgrados en identificar y definir indicadores bibliométricos apropiados que puedan incluir en los lineamientos para la elaboración de las tesis.

Por último y también no menos relevante es analizar si las tesis publicadas en el repositorio son realmente las tesis aprobadas ya que pueden estar en este repositorio tesis que en razón de agilizar el tiempo para su defensa, se entreguen, sin ser éste, el documento final y que esto último impida ser una evidencia importante del trabajo de investigación realizado.

Agradecimientos. Este trabajo forma parte del proyecto CSH043-15 Financiado por PAICYT-UANL. 


\section{Referencias}

Bordons, M., y Ángeles Zuluetab, M. A. (1999). Evaluación de la actividad científica a través de indicadores bibliométricos. Revista Española de Cardiología, 52(10), 790-800.

Brito Ocampo, L. B.; Ladrón de Guevara Solís, M., Rosas Poblano, S. G. (2010) Impacto de la biblioteca universitaria en los estudios de posgrado. Ibersid, 4, 195-199.

De-Miguel, Mario (2010). La evaluación de tesis doctorales. Propuesta de un modelo. Relieve, 16(1), 1-18.

García Córdoba, F. (1998). La tesis y el trabajo de tesis: Recomendaciones metodológicas para la elaboración de los trabajos de tesis. México: Editorial Spanta.

González de Requena, J. A., Andana, C., y Duhart, C. (2015). La condición intertextual en las citas de los artículos de investigación de psicología. Literatura y lingüística, 32, 181-200.

Hernández Sampieri, R., Fernández Collado, C., y Bautista Lucio, P. (2010). Metodología de la investigación ( $5^{a}$ ed.) México: Mc Graw-Hill.

Jiménez-Vásquez, M. S. (2014). Trayectorias profesionales de egresados del Doctorado en Educación de la Universidad Autónoma de Tlaxcala. Un análisis de las funciones, productividad y movilidad en el mercado académico. Perfiles educativos, 36(143), 30-48.

Meneses Aguirre, B., Romero Pedraza, E., y Sesma-Muñoz, B. (2013). Análisis y evolución de las referencias en las tesis de un posgrado en la Universidad Veracruzana, Ciencia Administrativa, 1, 34-38.

Musi-Lechuga, B. (2011). Análisis de la producción científica de los programas de doctorado de psicología con mención de calidad en España. (Tesis doctoral, Universidad de Granada).

Ortiz Torres, E., González Guitián, M. V., González Calzadilla, C., \& Infante Pérez, I. (2010). Evaluación del impacto científico de las tesis doctorales en Ciencias Pedagógicas mediante indicadores cienciométricos. Revista Española de Documentación Científica, 33(2), 279-286.

Osca-Lluch, J., Civera Mollá, C., y Peñaranda Ortega, M. (2009). Consecuencias de los errores en las referencias bibliográficas. El caso de la revista Psicothema. Psicothema, 21(2), 300-303.

Oyola-García, A. E., Soto-Cabezas, M. G., y Quispe-llanzo, M. P. (2014). La antigüedad de las referencias bibliográficas en publicaciones científicas. An Fac med. 75(4) 381.

Patiño Días, G. (2007). Citas y referencias bibliográficas. Bogotá: Universidad javeriana.

Pulgarín, A., Carapeto, C., \& Cobos, J.M. (2004). Análisis bibliométrico de la literatura científica publicada en Ciencia. Revista hispanoamericana de ciencias puras y aplicadas (1940-1974). Information Research, 9(4).

Retamozo, M. (2014). ¿Cómo hacer un proyecto de tesis doctoral en Ciencias Sociales? Ciencia, Docencia y Tecnología, 25(48), 173-202. 
Rivera Camino, J. (2011). Cómo escribir y publicar una tesis doctoral. Madrid: ESIC.

Sánchez Maríñez, J. (2008). Una propuesta conceptual para diferenciar los programas de postgrado profesionalizantes y orientados a la investigación. Implicaciones para la regulación, el diseño y la implementación de los programas de postgrado. Ciencia y Sociedad, 33(3), 327-341.

Secretaría de Educación Pública. (2013). Programa Sectorial de Educación 2013-2018.

Silva Hernández, D., Rodríguez Felizola, M. C., del Campo Peña, A. D. (2013). Las referencias bibliográficas en artículos originales y de revisión en revistas biomédicas cubanas, Revista Cubana de Salud Pública, 39(1), 83-95.

Torres-Velandia, S., Ruiz-Ávila, D., Meza-López, L. D. (2017). Infraestructura y equipamiento tecnológico en los doctorados consolidados de CONACyT: una mirada desde las políticas públicas y los académicos. Revista Iberoamericana de Educación Superior, 8(21), 3-23.

Urbano, C. (2000). Tipología documental citada en tesis doctorales de informática: bases empíricas para la gestión equilibrada de colecciones, Biblioteconomia i documentació, 5 .

Vallejo, M., Fernández, A., \& Torralbo, M. (2006). Patrones de citación en la investigación española en educación matemática. Revista Española de Documentación Científica, 29(3), 382-397.

Vallmitjana, N., \& Sabate, L. (2008). Citation analysis of Ph.D. dissertation references as a tool for collection management in an academic chemistry library. College \& Research Libraries, 69(1) 72-82. 